\title{
Le brome des prés
}





\section{Le brome des prés}

\section{R.P. Knowles}

Station de recherches

Saskatoon (Saskatchewan)

\section{V.S. Baron}

Station de recherches

Lacombe (Alberta)

\section{D.H. McCartney}

Station de recherches

Melfort (Saskatchewan)

\section{Illustration de la couverture}

Plant typique de brome des prés

\section{Agriculture Canada Publication 1889/F}

On peut se procurer des exemplaires à la

Direction générale des communications

Agriculture Canada, Ottawa (Ont.) K1A 0C7

CMinistre des Approvisionnements et Services Canada 1993

No de cat. A53-1889/1993F ISBN 0-662-97993-1

Impression 1993 2M-03:93

Production du Service aux programmes de recherches

Also available in English under the title of Meadow bromegrass 



\section{Table des matières}

Introduction 5

Description du brome des prés 5

Adaptation 7

Comparaison du rendement du brome des prés avec celui d'autres graminées 8

Cultivars de brome des prés 10

Comportement agronomique des cultivars 11

Établissement de peuplements 12

Utilisation comme pâture 13

Utilisation pour la production de foin 15

Qualité du fourrage 15

Production de semences 17

Lectures suggérées 20

Annexe 21 
Digitized by the Internet Archive in 2012 with funding from

Agriculture and Agri-Food Canada - Agriculture et Agroalimentaire Canada 


\section{Introduction}

Le brome des prés, Bromus riparius Rehm. (aussi appelé $B$. erectus Huds.), a un comportement traçant, des rhizomes courts et une bonne capacité de repousse. Il croît naturellement dans le sud-est de l'Europe, dans le Caucase, en Turquie et en Asie centrale. L'enregistrement du cultivar Regar aux États-Unis en 1966 marque les débuts de sa culture en Amérique du Nord. Ce cultivar, dérivé d'une plante introduite de la Turquie, a été enregistré au Canada en 1980, et est utilisé depuis avec succès, comme culture de pâture, en Alberta et en Saskatchewan. En 1987, la Station fédérale de recherches agricoles de Saskatoon (Sask.) a mis sur le marché le Paddock et le Fleet, des cultivars au rendement supérieur en semences.

Dans la présente brochure, nous comparons le brome des prés au brome inerme, $B$. inermis Leyss., cultivé à plus grande échelle. Nous soulignons en particulier les qualités du brome des prés en tant que culture de pâture. Les stations de recherches d'Agriculture Canada situées en Alberta et en Saskatchewan ont fourni des données sur le rendement, tandis que les éleveurs de bovins et les semenciers de ces provinces ont fourni d'autres observations.

\section{Description du brome des prés}

Les plants de brome des prés portent de nombreuses feuilles basales (voir l'illustration sur la couverture), une caractéristique qui, conjuguée à une repousse rapide après le broutage, rend cette graminée utile pour

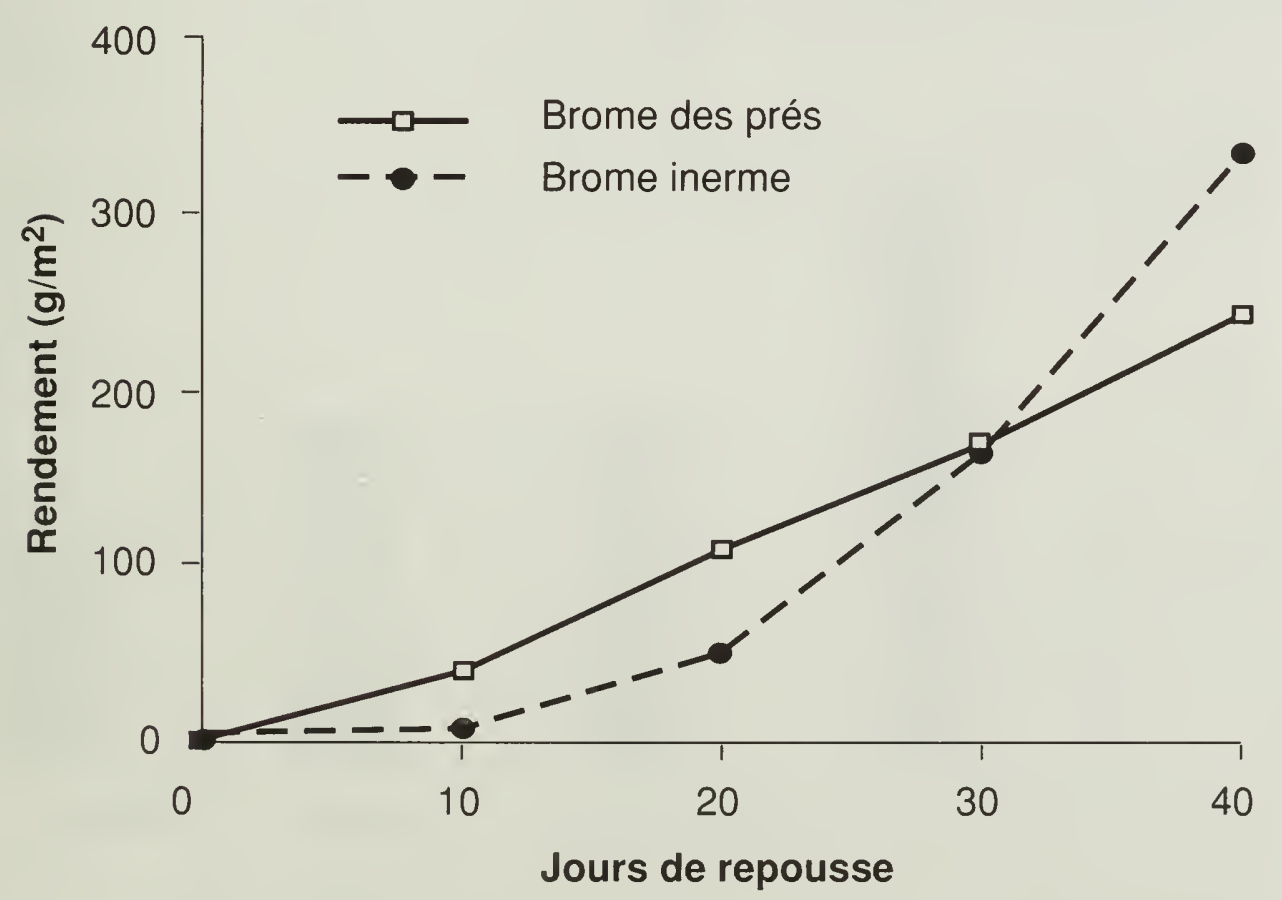

Fig. 1 Rendement cumulatif en matière sèche du brome des prés et du brome inerme obtenu au cours d'essais en serre, à Lacombe (Alb.). 
la pâture. Le regain est particulièrement rapide au début (de 0 à 20 jours) comparativement au brome inerme (fig. 1). En effet, la repousse s'amorce à partir de la base des talles existantes tandis que, chez le brome inerme, elle commence sous terre, à partir des couronnes et des rhizomes. Lorsqu'elles sont coupées ou broutées fréquemment, les talles du brome inerme ne parviennent jamais à une croissance rapide; par contre, celles du brome des prés réussissent à se développer rapidement.

Pendant la campagne, la production du brome des prés est plus uniforme que celle du brome inerme, et lui est particulièrement supérieure en juillet et en septembre (fig. 2). De plus, ses feuilles résistent mieux à la gelée que celles du brome inerme; cette graminée peut donc être broutée jusqu'à la mi-octobre pour autant que les conditions d'humidité soient favorables.

Les rhizomes du brome des prés sont beaucoup plus courts que ceux du brome inerme. À Saskatoon, les plants d'un an avaient en moyenne un diamètre de $42 \mathrm{~cm}$, comparativement à $77 \mathrm{~cm}$ dans le cas du brome inerme.

Les feuilles du brome des prés sont plus étroites que celles de l'autre espèce et sont pubescentes; leurs petits poils sont particulièrement visibles sur les bords (fig. 3). En outre, elles ont tendance à retomber et certaines d'entre elles peuvent être perdues parce qu'elles ont été piétinées par le bétail. Les tiges et les graines sont également plus ou moins pubescentes. Les graines du brome des prés sont garnies de courtes barbes mesurant de 5 à $8 \mathrm{~mm}$ de longueur, contre au plus $2 \mathrm{~mm}$

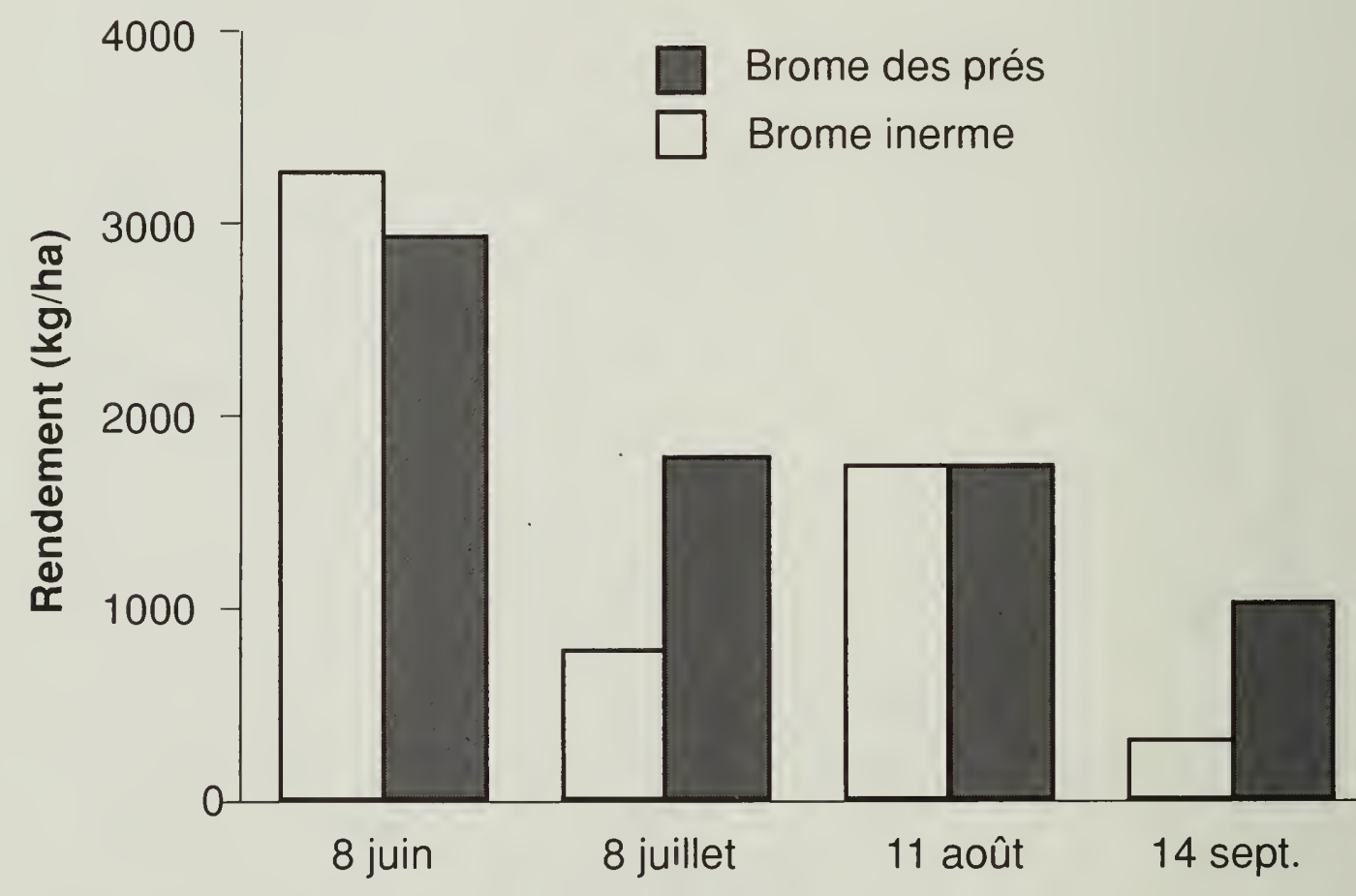

Fig. 2 Distribution du rendement du brome des prés en comparaison avec le brome inerme au cours de coupes répétées effectuées quatre fois par saison, à Lacombe (Alb.). 


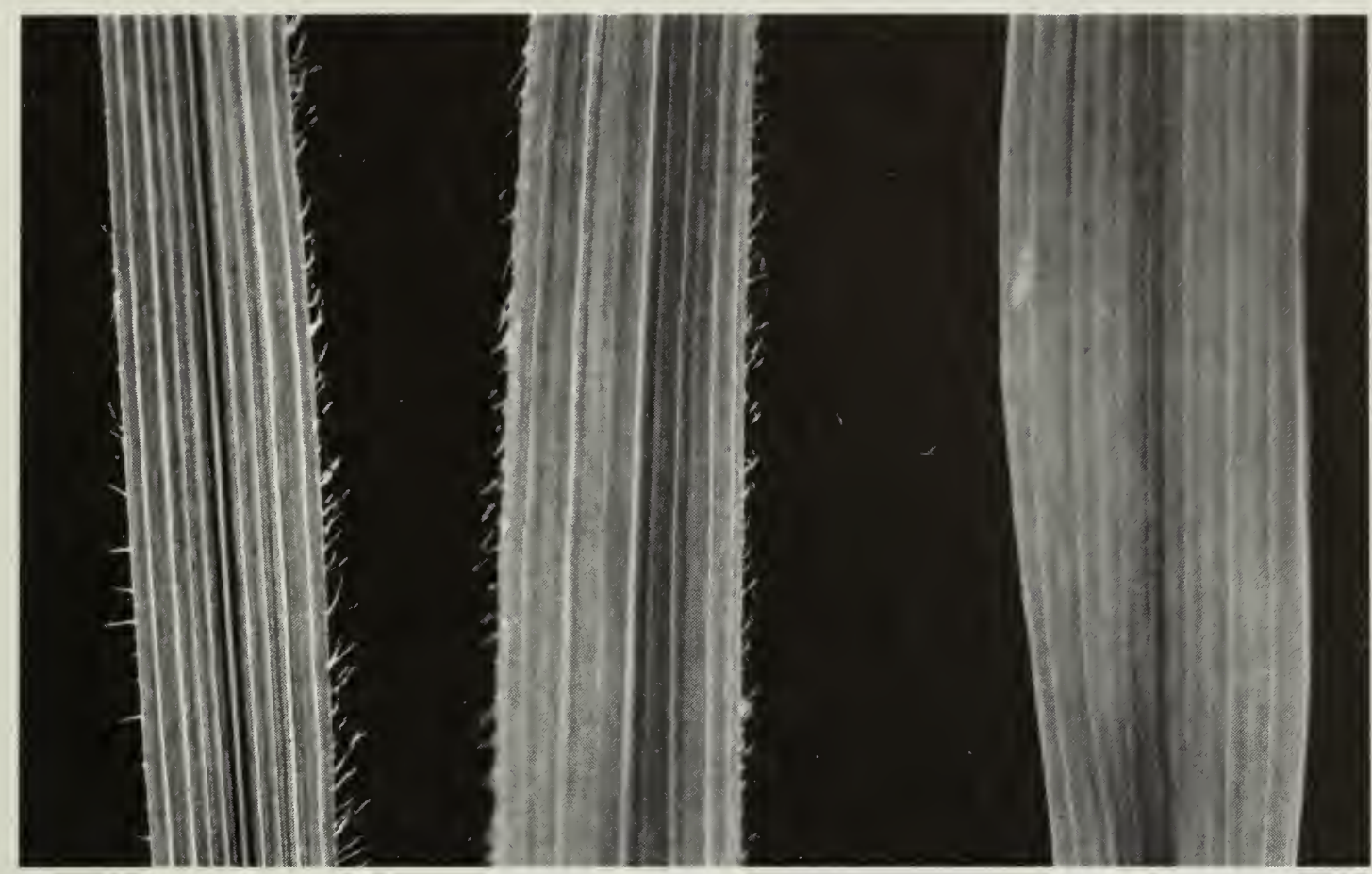

Fig. 3 Feuilles du brome des prés (gauche), du brome inerme (droite) et de leur hybride (centre) montrant la pubescence typique et la largeur relative des feuilles.

dans le cas des barbes apicales du brome inerme. Le poids de 1000 graines varie entre 5 et $6 \mathrm{~g}$ pour le brome des prés, et entre 3 et $4 \mathrm{~g}$ pour le brome inerme.

Les deux graminées sont diploides et comptent 70 chromosomes dans le cas du brome des prés et 56 pour ce qui est du brome inerme. Toutes deux sont allogames, mais l'autofertilité du brome des prés est supérieure à celle de l'autre espèce.

Le brome des prés résiste à la tache helminthosporienne Pyrenophora bromi Died., qui cause de lourdes pertes chez le brome inerme, en particulier dans les peuplements établis peu fertiles.

\section{Adaptation}

Le brome des prés convient le mieux aux zones plus froides et plus humides de l'aire d'adaptation plus étendue du brome inerme, notamment les zones de sols noirs et de sols boréals lessivés, ainsi que les districts plus humides des zones de sols brun foncé. Grâce à l'irrigation, il a également produit de bons rendements dans la zone des sols bruns. Sa production est bonne dans les sables, les loams et les argiles.

A Saskatoon, le brome des prés s'est maintenu au moins 10 ans avec un désherbage minimal. Des essais ont montré qu'il persiste mieux que le brome inerme dans les sols sablonneux pauvres soumis à un broutage intensif. En périodes de sécheresse, les feuilles du brome des prés brunissent plus rapidement que celles de l'autre espèce, sans menacer toutefois la survie de la plante. 
Le brome des prés est également sensible aux inondations et périra s'il est inondé pendant 10 jours ou plus. Il tolère moins bien la salinité que le brome inerme. Lorsqu'on cultive le brome des prés sur un terrain vallonné, il est recommandé de procéder au sursemis des zones mouillées et basses avec des espèces plus tolérantes comme la fléole des prés, dans les endroits humides, et le brome inerme, dans les endroits plus secs.

Le brome des prés est moins rustique que le brome inerme; en effet, $50 \%$ des plaques herbacées de cette espèce subissent des dommages à des températures de $22{ }^{\circ} \mathrm{C}$ sous zéro, alors que le mercure doit baisser de 29 à $35{ }^{\circ} \mathrm{C}$ sous zéro pour observer des dommages équivalents chez le brome inerme et l'agropyre à crête, respectivement (Limin et Fowler, 1987).

\section{Comparaison du rendement du brome des prés avec celui d'autres graminées}

Au cours d'essais effectués sur des sols noirs, à Melfort, et sur des sols boréals lessivés, près de Melfort, on a comparé le rendement du brome des prés à celui de cinq autres graminées, dont le brome inerme (tableaux 1 et 2). Dans les prairies à pâturer qui subissent quatre coupes, le rendement du brome des prés a dépassé celui des autres espèces sur les deux types de sol. Dans les champs de fauche soumis à deux coupes, le brome des prés a donné des rendements supérieurs à ceux de toutes les autres espèces, sauf l'agropyre à crête sur les sols boréals lessivés. Cependant, le rendement a été inférieur par rapport à toutes les autres espèces, à l'exception de la stipe verte, sur les sols noirs. Sur les sols noirs humides de Lacombe, le brome des prés a affiché un rendement fourrager de $20 \%$ supérieur au rendement moyen de la stipe verte, du brome inerme, du dactyle pelotonné et du vulpin

Tableau 1 Rendement d'espèces de graminées sur des sols fertilisés annuellement, à raison de $90 \mathrm{~kg}$ d'azote à l'hectare et de $22 \mathrm{~kg}$ de phosphore à l'hectare, sous un régime pastoral simulé à Melfort (Sask.)

\begin{tabular}{lcc}
\hline & \multicolumn{2}{c}{$\begin{array}{c}\text { Rendement annuel en matière } \\
\text { sèche pour quatre coupes (kg/ha) }\end{array}$} \\
\cline { 2 - 3 } Espèce (cultivar) & $\begin{array}{c}\text { Sols noirs } \\
1980-1986\end{array}$ & $\begin{array}{c}\text { Sols boréals lessivés } \\
1981-1986\end{array}$ \\
\hline Brome des prés (Regar) & 5432 & 2063 \\
Brome inerme (Carlton, Magna*) & 5070 & 1881 \\
Agropyre à crête (Parkway) & 5304 & 1896 \\
Agropyre intermédiaire (Chief) & 5994 & 1882 \\
Élyme de Russie (Mayak) & 4932 & - \\
Stipe verte (Lodorm) & 4987 & 1807 \\
\hline
\end{tabular}

* Magna cultivé sur des sols boréals lessivés seulement. 
Tableau 2 Rendement en foin d'espèces de graminées sur des sols fertilizés annuellement, à raison de $90 \mathrm{~kg}$ d'azote à l'hectare et de $22 \mathrm{~kg}$ de phosphore à l'hectare, à Melfort (Sask.)

Rendement annuel en matière sèche pour deux fauches $(\mathrm{kg} / \mathrm{ha})$

Espèce (cultivar)

Sols noirs

1980-1986

Sols boréals lessivés

1981-1986

Brome des prés (Regar)

Brome inerme (Carlton, Magna*)

Agropyre à crête (Parkway)

Agropyre intermédiaire (Chief)

Élyme de Russie (Mayak)

Stipe verte (Lodorm)

$\begin{array}{lr}6251 & 2581 \\ 7619 & 2457 \\ 7957 & 2684 \\ 7166 & 2192 \\ 6658 & - \\ 6069 & 2220\end{array}$

* Magna cultivé sur des sols boréals lessivés seulement.

des prés (tableau 3). Le brome des prés a donné du fourrage prêt à être pâturé à une date aussi précoce que le vulpin des prés. Sa production automnale et sa repousse totale ont été semblables à celles du dactyle pelotonné. De même, sur les sols noirs de Beaverlodge, le brome des prés a mieux résisté aux fréquentes défoliations que le brome inerme; en outre, sa production a été supérieure dans des conditions de faible taux de fertilisation en azote (Fairey, 1991).

À Saskatoon, dans la zone de sols brun foncé plus secs, le brome des prés, cultivé pour la pâture ou pour la fauche, a produit moins que le brome inerme (tableau 4). À Scott, les deux graminées ont donné des rendements

Tableau 3 Rendement du brome des prés utilisé comme pâture comparé à celui d'autres graminées, à Lacombe (Alb.), pour quatre coupes par saison*, de 1988 à 1991

\begin{tabular}{llc}
\hline Espèce & Cultivar & Rendement moyen total $(\mathrm{kg} / \mathrm{ha})$ \\
\hline Brome des prés & Regar & 12114 \\
& Paddock & 11702 \\
\multirow{2}{*}{ Brome inerme } & Fleet & 11972 \\
& Carlton & 9648 \\
Agropyre à crête & Manchar & 9710 \\
& Kirk & 10095 \\
Dactyle pelotonné & Parkway & 10332 \\
Vulpin des prés & Kay & 9759 \\
& Commun & 10284 \\
\hline
\end{tabular}

* Sols fertilisés après chaque coupe, à raison de $244 \mathrm{~kg} / \mathrm{ha}$ de $\mathrm{N}$ et $63 \mathrm{~kg} / \mathrm{ha}$ de $\mathrm{P}$ par année. 
Tableau 4 Rendement d'espèces de graminées utilisées pour la production de foin et comme pâture, à Saskatoon et à Scott (Sask.), sur des sols brun foncé sans fertilisation

\begin{tabular}{|c|c|c|c|c|}
\hline \multirow[b]{3}{*}{ Espèce (cultivar) } & \multicolumn{4}{|c|}{ Rendement annuel (kg/ha) } \\
\hline & \multicolumn{2}{|c|}{$\begin{array}{l}\text { Saskatoon, } \\
1975-1983\end{array}$} & \multicolumn{2}{|c|}{$\begin{array}{c}\text { Scott, } \\
1975-1980\end{array}$} \\
\hline & Foin & Pâture & Foin & Pâture \\
\hline Brome des prés (Paddock) & 2082 & 1996 & 2859 & 1398 \\
\hline Brome inerme (Carlton) & 2841 & 2178 & 2834 & 1608 \\
\hline Agropyre à crête (Fairway) & 2218 & 1466 & 2548 & 1672 \\
\hline Agropyre intermédiaire (Chief) & 3819 & 2534 & 3270 & 1585 \\
\hline Élyme de Russie (Mayak) & 2311 & 1941 & 2633 & 1388 \\
\hline
\end{tabular}

Source : Knowles, 1987.

équivalents lorsque cultivées pour la fauche, quoique le brome inerme ait fourni un rendement supérieur comme pâture. Il est avantageux de cultiver le brome des prés dans cette zone parce qu'il repousse plus rapidement après la coupe et qu'il reste plus verdoyant ou de meilleure qualité à l'automne. En outre, on ne pouvait obtenir que deux ou trois coupes comparativement à quatre dans la zone des sols noirs.

\section{Cultivars de brome des prés}

Le cultivar Regar est utilisé avec succès dans les prairies irriguées de l'Idaho, du Wyoming et du Montana. Il donne également un bon rendement en Alberta et en Saskatchewan. Son principal défaut est de produire peu de graines ce qui se traduit par des prix élevés pour les semences.

Le cultivar Paddock provient d'une plante de Krasnodar (URSS) introduite à la Station de recherches de Saskatoon en 1969. Il a été enregistré en 1987, et sa distribution a été confiée en exclusivité par l'Association SeCan à la Newfield Seeds Ltd., de Nipawin (Sask.).

Également mis au point à la Station de recherches de Saskatoon, le cultivar Fleet a été homologué en 1987. Il est issu de l'interpollinisation de huit souches européennes et asiatiques, y compris des plantes de Regar et de Paddock. La sélection a été exercée particulièrement en faveur des plantes affichant une bonne production semencière, aux barbes peu développées et plus résistantes à l'égrenage prématuré. L'Association SeCan a mis cette variété à la disposition générale de ses membres aux fins de production de semences contrôlées et de fondation. En comparaison avec le Regar, les cultivars canadiens Paddock et Fleet ont une production semencière beaucoup plus élevée et un rendement en fourrage équivalent. 


\section{Comportement agronomique des cultivars}

Les trois cultivars du brome des prés ont affiché des rendements assez semblables dans le cadre d'essais menés sur leur rendement en pâturage, établi à l'aide d'une coupe partielle effectuée en mai et de coupes partielles mensuelles par la suite (tableau 5). Dans des conditions plus sèches, à Saskatoon, le brunissement des feuilles était plus prononcé chez le Regar que chez les cultivars Paddock et Fleet. À Lacombe, où les conditions étaient plus humides, le cultivar Regar a fait preuve d'une meilleure vigueur printanière.

Les rendements en foin, repousse comprise, ont été semblables pour les trois cultivars (tableau 6) bien que le cultivar Fleet n'ait été soumis à des essais qu'à un seul des quatre emplacements.

$\mathrm{Au}$ cours des essais sur le rendement en semences effectués à trois endroits, les cultivars Paddock et Fleet ont montré une production bien meilleure que celle du cultivar Regar (tableau 7). Les troisième et quatrième années, le rendement a été plus soutenu dans le cas du Paddock et du Fleet que dans celui du Regar (tableau 8). En général, le rendement en semences du brome des prés fléchissait davantage avec l'augmentation du nombre des années de production que celui du brome inerme.

Tableau 5 Rendement de cultivars de brome des prés utilisés comme pâture soumis à des coupes partielles

Rendement annuel (kg/ha)

\begin{tabular}{lccrr} 
Station (essais) & Années & Paddock & Fleet & Regar \\
\hline Saskatoon, Sask. (6 essais) & $1980-1987$ & 3278 & 3265 & 2994 \\
Lacombe, Alb. (3 essais) & $1981-1988$ & 8656 & - & 8411 \\
Brandon, Man. (1 essai) & $1982-1983$ & 5960 & 5895 & 6130 \\
Lethbridge, Alb. (1 essai*) & $1986-1988$ & - & 10900 & 10900
\end{tabular}

* Sols irrigués; les autres essais ont été effectués sur des sols non irrigués.

Tableau 6 Rendement en foin de cultivars de brome des prés, repousse comprise

Rendement annuel (kg/ha)

$\begin{array}{lcrrr}\text { Station (essais) } & \text { Années } & \text { Paddock } & \text { Fleet } & \text { Regar } \\ & & & - & 5514 \\ \text { St. Claude, Man. (3 essais) } & 1982-1986 & 5832 & - & 3601 \\ \text { Saskatoon, Sask. (2 essais) } & 1981-1990 & 3729 & 3679 & 5401 \\ \text { Melfort, Sask. (1 essai) } & 1979-1986 & 5408 & - & 12632 \\ \text { Lacombe, Alb. (2 essais) } & 1983-1988 & 13084 & - & \end{array}$


Tableau 7 Rendement en semences de cultivars de brome des prés

\begin{tabular}{lcccc}
\hline & & \multicolumn{3}{c}{ Rendement annuel (kg/ha) } \\
\cline { 3 - 5 } Station (essais) & Années & Paddock & Fleet & Regar \\
\hline Saskatoon, Sask. (5 essais) & $1981-1989$ & 379 & 447 & 210 \\
Melfort, Sask. (1 essai) & $1985-1988$ & 351 & 360 & 229 \\
Arborg, Man. (3 essais) & $1982-1988$ & 663 & - & 417 \\
\hline
\end{tabular}

Tableau 8 Rendement en semences de cultivars de brome des prés obtenu à Saskatoon, par année de récolte, avec de grands $(90 \mathrm{~cm})$ et de petits $(30 \mathrm{~cm})$ espacements, dans des prairies ensemencées en 1984 et fertilisées annuellement à raison de $50 \mathrm{~kg} / \mathrm{ha}$ de $\mathrm{N}$

\begin{tabular}{|c|c|c|c|c|c|}
\hline \multirow[b]{2}{*}{ Cultivar } & \multicolumn{5}{|c|}{ Rendement en semences $(\mathrm{kg} / \mathrm{ha})$} \\
\hline & 1985 & 1986 & 1987 & 1989 & Moyenne** \\
\hline & \multicolumn{5}{|c|}{ Grands espacements } \\
\hline Regar & 179 & 494 & 301 & 154 & 282 \\
\hline Paddock & 310 & 818 & 543 & 427 & 524 \\
\hline Fleet & 295 & 798 & 661 & 491 & 561 \\
\hline \multirow[t]{2}{*}{ Carlton* } & 233 & 608 & 488 & 559 & 472 \\
\hline & \multicolumn{5}{|c|}{ Petits espacements } \\
\hline Regar & 378 & 283 & 55 & 50 & 192 \\
\hline Paddock & 716 & 438 & 126 & 98 & 345 \\
\hline Fleet & 702 & 530 & 197 & 172 & 400 \\
\hline Carlton* & 416 & 480 & 309 & 261 & 367 \\
\hline
\end{tabular}

* Utilisation du brome inerme comme témoin.

*** Ne comprend pas l'année 1988, année où la sécheresse a empêché toute production de semences.

\section{Établissement de peuplements}

Les producteurs ont trouvé qu'il était plus difficile d'établir des peuplements de brome des prés que des peuplements de brome inerme. Cette difficulté est en partie liée au fait que le brome des prés n'a pas un comportement traçant aussi prononcé que le brome inerme; les peuplements ne "s'étalent » donc pas. Les graines du brome des prés étant plus grosses que celles du brome inerme, il faut donc augmenter la densité de semis. En outre, à moins que les barbes apicales et les poils des graines ne soient éliminées, les graines forment une voûte dans le semoir. 
La densité de semis recommandée pour le brome des prés est de $12 \mathrm{~kg} / \mathrm{ha}$ en culture simple ou mixte. En culture mixte, semer aussi de la luzerne à raison de 1 à $3 \mathrm{~kg} / \mathrm{ha}$. Dans les régions plus humides, réduire la densité de semis de la luzerne de 0,5 à $0,75 \mathrm{~kg} / \mathrm{ha}$ pour diminuer la proportion de cette espèce et, ainsi, le danger de météorisation. Comme espèces de remplacement pour la culture mixte, mentionnons le lotier corniculé, à raison de $2 \mathrm{~kg} / \mathrm{ha}$, ou l'astragale pois chiche, à une densité de 5 à $6 \mathrm{~kg} / \mathrm{ha}$, deux légumineuses qui ne provoquent pas de météorisation.

Dans les peuplements prévus pour la pâture et la fauche, adopter une interligne de 15 à $30 \mathrm{~cm}$. Pour la production de semences, utiliser des écarts de 30,60 et $90 \mathrm{~cm}$.

Les céréales semées couramment comme culture-abri la première année réduisent de beaucoup la vigueur du brome des prés dans l'année qui suit son ensemencement. Cette perte de vigueur retarde le broutage l'année suivante jusqu'à ce que la graminée soit bien établie. Pour la production de semences, il est recommandé de semer le brome des prés sans culture-abri.

\section{Utilisation comme pâture}

Les bovins acceptent bien le brome des prés. Ils le broutent sélectivement dans des peuplements où il est mélangé à d'autres espèces de plantes fourragères, bien qu'ils puissent le rejeter un peu au début jusqu'à ce qu'ils s'y habituent. Lorsque le brome des prés est laissé sans être brouté jusqu'à la formation du grain, son appétibilité est réduite, mais pas autant que celle de l'agropyre à crête et du brome inerme. À des taux de chargement de faibles à modérés, le broutage peut ne pas être uniforme, ce qui entraînera le surpâturage ou le pâturage insuffisant de certains plants. On a signalé que les moutons préfêrent le brome inerme au brome des prés.

Le brome des prés a un type de croissance qui le rend propice à un broutage de grande intensité sur de courtes périodes. Au moyen de clôtures installées à l'intérieur d'enclos, on peut créer plusieurs enclos, lesquels peuvent être soumis au broutage, puis laissés au repos afin de permettre au brome des prés de repousser avant d'être brouté de nouveau. Il faut attendre que le brome des prés ait atteint une hauteur de 20 à $25 \mathrm{~cm}$ avant de l'utiliser comme pâture. La densité des animaux mis à l'herbe doit être suffisante pour que la graminée soit ramenée rapidement à une hauteur de $10 \mathrm{~cm}$, de préférence en 7 à 12 jours. Lorsque la période de broutage est plus longue, les animaux commencent à brouter la repousse, ce qui affaiblit les plants. Entre les périodes de broutage, il faut laisser le pâturage au repos pendant 30 à 60 jours, selon la saison et l'importance des précipitations. En ayant recours au pâturage en rotation, il est possible d'obtenir deux à trois broutages par année. Faire en sorte que les animaux broutent le pâturage de brome des prés pour en rabaisser la hauteur à $10 \mathrm{~cm}$ avant la neige afin d'empêcher l'enchevêtrement des feuilles propice aux maladies. 
Le brome des prés peut être considéré comme une culture fourragère à double usage : la pâture et la fauche. À la fin de juin, on peut faucher les plants dans les enclos qui n'ont pas servi de pâturage au printemps, puis les laisser repousser pour le broutage à l'automne. Les plants des enclos soumis à un broutage léger au début de juin pourront de nouveau être broutés ou être fauchés à la fin de juillet ou au début d'août.

Il est rare que le brome des prés produise des tiges porte-graines lorsqu'il repousse après un premier broutage complet. La production de tiges porte-graines est également beaucoup moindre dans les peuplements établis depuis longtemps et dont la fertilité est réduite. Un broutage léger au printemps réduit la formation de tiges portegraines et stimule une plus grande production de feuilles.

Le brome des prés commence à croître tôt au printemps et il est prêt à être brouté 10 jours avant le brome inerme. L'agropyre à crête peut être brouté entre 5 et 7 jours avant le brome des prés; cette espèce est donc plus propice à un broutage printanier très précoce; elle tolère en outre davantage un broutage printanier important et intensif. Le brome des prés convient particulièrement bien au broutage à l'automne parce qu'il pousse bien par temps frais. Utilisé de façon complémentaire avec d'autres graminées cultivées et indigènes, le brome des prés peut servir de pâture à la fin de l'été et à l'automne, une période où d'autres graminées, comme le brome inerme, sont moins productives.

Dans les nouveaux peuplements fertilisés, une unité animale-mois (UAM) peut n'avoir besoin que de 0,12 ha pour le broutage, contre 0,24 ha dans le cas des peuplements établis depuis longtemps. Les gains enregistrés par les animaux ont varié entre 0,72 et $0,86 \mathrm{~kg} / \mathrm{jour}$, selon la fertilité du sol, les précipitations et la conduite de l'élevage. À la Station de recherches de Melfort, on a comparé les gains des animaux mis en pâturage continu dans des prairies de brome des prés et de brome inerme (tableau 9). Ces gains ont été comparables pour les deux graminées du 15 juin au 11 août, mais clairement supérieurs dans le cas du brome des prés du 11 août au 15 octobre.

Tableau 9 Gains de poids vif observés chez des génisses broutant du brome des prés et du brome inerme, à Melfort (Sask.), 1987

Gains quotidiens de poids vif ( $\mathrm{kg} / \mathrm{ha})$

Période de broutage

Brome des prés

Brome inerme

Du 15 juin au 14 juillet

Du 14 juillet au 11 août

Du 11 août au 18 septembre

6,9

6,7

3,5

3,5

Du 18 septembre au 5 octobre

Total (kg/ha)

3,7

2,9

1,4

458

0,3

404 


\section{Utilisation pour la production de foin}

Sous un régime de fauche, le rendement du brome des prés est habituellement moins élevé que celui du brome inerme. Cependant, après avoir été fauché, le brome des prés récupère mieux et fournit des pâturages à la fin de l'été et à l'automne. Ce double usage du brome des prés convient mieux aux régions où les précipitations sont plus abondantes.

Pour ce qui est de son utilisation en culture mixte avec la luzerne pour la production de foin, la proportion de luzerne semble dépendre des précipitations. Dans des conditions de faibles précipitations, à Saskatoon, le brome des prés maintient un meilleur équilibre avec la luzerne comparativement au brome inerme. Dans des conditions plus humides, à Lacombe, le brome des prés livre une concurrence vive à la luzerne. Sous un régime de fauche pâture, la repousse rapide du brome des prés dans les mélanges avec la luzerne est souhaitable parce qu'elle permet un meilleur équilibre entre la graminée et la luzerne et réduit ainsi le danger de météorisation.

\section{Qualité du fourrage}

La qualité fourragère du brome des prés est à peine inférieure à celle du brome inerme (tableau 10). À la Station de recherches de Lacombe, ori a réalisé des analyses complètes de la valeur nutritive à quatre stades de la croissance initiale et à trois stades de la repousse automnale. Aux stades de la croissance initiale au printemps et du pré-bouton, les teneurs en protéines et en fibres du brome des prés étaient légèrement plus faibles et plus élevées que celles du brome inerme (tableau 10). Ces différences se sont atténuées aux stades de la préanthèse et de la floraison (foin). Les différences dans la digestibilité in vitro entre le brome des prés et le brome inerme étaient faibles aux trois premiers stades mais, à la floraison, le brome des prés était plus digestible. D'après les résultats, il serait plus avantageux de mettre en réserve le brome des prés en vue d'une utilisation ultérieure qu'il ne le serait pour le brome inerme.

En août et en septembre, la repousse du brome des prés avait de nouveau une teneur en protéines légèrement inférieure et une teneur en fibres légèrement supérieure à celles du brome inerme (tableau 11). La digestibilité des deux espèces était comparable. Malgré la qualité légèrement moindre du brome des prés aux stades d'utilisation comme pâture, sa valeur nutritive ne semblerait pas limiter la production de veaux d'embouche ni de bovins de long engraissement. 
Tableau 10 Qualité fourragère du brome des prés et du brome inerme à quatre stades de la croissance initiale, à Lacombe (Alb.), 1987-1989

\begin{tabular}{|c|c|c|c|c|}
\hline \multirow[b]{2}{*}{ Espèce } & \multicolumn{4}{|c|}{ Stades de croissance* } \\
\hline & $\begin{array}{c}\text { Période } \\
\text { végétative }\end{array}$ & Pré-bouton & Préanthèse & Floraison \\
\hline - & \multicolumn{4}{|c|}{ Digestibilité in vitro (\%) } \\
\hline \multirow{3}{*}{$\begin{array}{l}\text { Brome des prés } \\
\text { Brome inerme }\end{array}$} & 78,6 & 75,5 & 66,2 & 63,8 \\
\hline & 78,4 & 76,1 & 66,1 & 58,1 \\
\hline & \multicolumn{4}{|c|}{ Protéines brutes (\%) } \\
\hline \multirow{3}{*}{$\begin{array}{l}\text { Brome des prés } \\
\text { Brome inerme }\end{array}$} & 24,7 & 19,3 & 13,8 & 11,6 \\
\hline & 27,2 & 21,3 & 15,4 & 12,2 \\
\hline & \multicolumn{4}{|c|}{ Cellulose au détergent neutre (\%) } \\
\hline Brome des prés & 51,2 & 57,8 & 66,8 & 64,0 \\
\hline \multirow[t]{2}{*}{ Brome inerme } & 49,0 & 53,8 & 65,9 & 64,5 \\
\hline & \multicolumn{4}{|c|}{ Fibres au détergent acide (\%) } \\
\hline Brome des prés & 21,2 & 24,3 & 33,0 & 32,3 \\
\hline \multirow[t]{2}{*}{ Brome inerme } & 19,2 & 22,0 & 31,8 & 33,4 \\
\hline & \multicolumn{4}{|c|}{ Lignine (\%) } \\
\hline Brome des prés & 2,2 & 2,2 & 3,8 & 3,3 \\
\hline Brome inerme & 1,9 & 1,9 & 3,8 & 3,6 \\
\hline
\end{tabular}

* Période végétative : La période végétative et le stade pré-bouton correspondent à la période du premier broutage de l'année; la préanthèse et la floraison représentent les stades de la fenaison. 
Tableau 11 Qualité fourragère de la repousse du brome des prés et du brome inerme en août et en septembre après la coupe partielle du $1^{\mathrm{er}}$ août, à Lacombe (Alb.), 1987-1989

Nbre de semaines après la coupe partielle

Espèce

2

4

6

Digestibilité in vitro de la matière organique (\%)

Brome des prés

Brome inerme

Brome des prés

Brome inerme

Brome des prés

Brome inerme

Brome des prés

Brome inerme

Brome des prés

Brome inerme

\section{5,1}

76,1
72,4

73,3

72,9

72,6

Protéines brutes (\%)

\begin{tabular}{lll}
\hline 25,0 & 19,9 & 17,9 \\
28,5 & 23,8 & 20,8
\end{tabular}

Cellulose au détergent neutre (\%)

\begin{tabular}{lll}
\hline 61,8 & 61,2 & 57,6 \\
54,3 & 58,5 & 56,2
\end{tabular}

Fibres au détergent acide (\%)

$\begin{array}{lll}26,6 & 28,3 & 27,1 \\ 22,2 & 24,0 & 23,5\end{array}$

Lignine (\%)

\begin{tabular}{lll}
\hline 1,8 & 2,8 & 2,2 \\
2,1 & 2,0 & 2,1
\end{tabular}

\section{Production de semences}

Le rendement semencier du brome des prés est moins uniforme que celui du brome inerme. Le cultivar Regar, en particulier, affiche un rendement en semences faible qui baisse rapidement après deux récoltes (tableau 8). Les cultivars Paddock et Fleet ont donné des rendements de $400 \mathrm{~kg} / \mathrm{ha}$ jusqu'à la quatrième récolte selon des interlignes de $90 \mathrm{~cm}$. Lorsque l'interligne était réduite à $30 \mathrm{~cm}$, le rendement en semences était élevé aux deux premières récoltes, puis fléchissait les troisième et quatrième années.

En 1991, à la suite d'un automne très sec l'année précédente, dans presque tous les champs de brome des prés de la Saskatchewan, la production de tiges porte-graines a été faible comparativement au brome inerme. Seuls les nouveaux peuplements et les champs irrigués ont donné une production normale de semences. 
Lorsque le brome des prés est cultivé pour la production de semences, il est suggéré de le semer au printemps dans des champs vierges, sans culture-abri. Cette méthode favorise au maximum la tendance de la culture à donner les meilleurs rendements au cours des première et deuxième années de production. Les producteurs ont utilisé des interlignes de 30,60 et $90 \mathrm{~cm}$ pour la production de semences. Lorsque les interlignes sont larges, il est bon de travailler le sol au printemps et à l'automne pour réduire la quantité de mauvaises herbes et de plantes spontanées.

Une certaine perte de rendement en semences chez le brome des prés peut être attribuable à la présence " d'épis argentés " (" coulure des graminées "), une condition qui influence plusieurs espèces de graminées, à l'exception généralement du brome inerme. À Saskatoon, jusqu'à $20 \%$ des panicules étaient ainsi endommagées à partir de la deuxième année de récolte des semences du brome des prés.

Le charbon de l'épi a réduit considérablement la production de semences chez le brome des prés (fig. 4). La maladie est causée par l'infection des semences utilisées pour établir le peuplement. Des pertes importantes sont généralement signalées la première année de récolte des semences, l'une des années les plus productrices d'habitude. Plus tard, les cultures semencières montrent moins de dommages, car les plants non touchés dominent. Il faut faire vérifier toute semence soupçonnée d'être porteuse du charbon et qui est destinée à la production de semences pour y déceler la présence de spores du charbon (consulter l'annexe).

L'égrenage prématuré est plus commun chez le brome des prés que chez le brome inerme. Malgré ce risque, certains producteurs préfêrent procéder au moissonnage-battage de la récolte et utiliser ensuite le chaume comme aliment pour animaux ou pour la pâture.

Les graines du brome des prés nécessitent un traitement plus poussé que celles du brome inerme. Le traitement consiste dans l'élimination des poils et des barbes apicales, ce qui améliore l'écoulement des graines dans les semoirs (fig. 5).

L'Association canadienne des producteurs de semences se préoccupe de l'hybridation possible du brome des prés avec le brome inerme cultivés dans des prairies adjacentes. Au cours d'expériences réalisées récemment à la Station de recherches de Saskatoon, on n'a pas obtenu de preuve d'intercroisement lorsque des graines provenant de prairies adjacentes ont été utilisées dans des tests sur la descendance. Bien que l'on puisse obtenir $20 \%$ d'hybridation dans des croisements contrôlés effectués en serre, l'hybridation ne semble pas se produire dans le champ. Le fait que le brome des prés fleurit 6 à 10 jours avant le brome inerme semble en être la raison. 


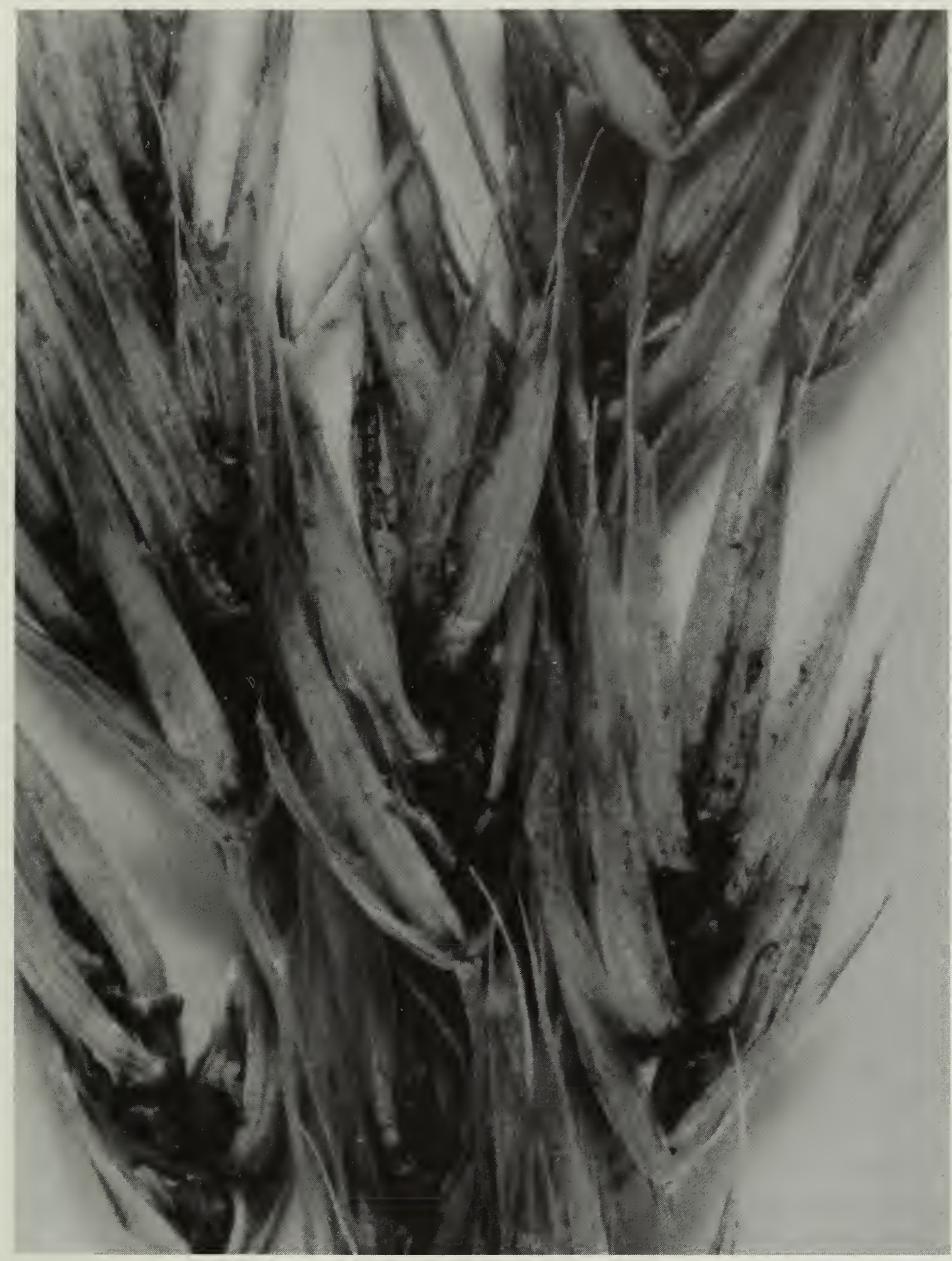

Fig. 4 Charbon de l'épi attaquant la panicule du brome des prés. 


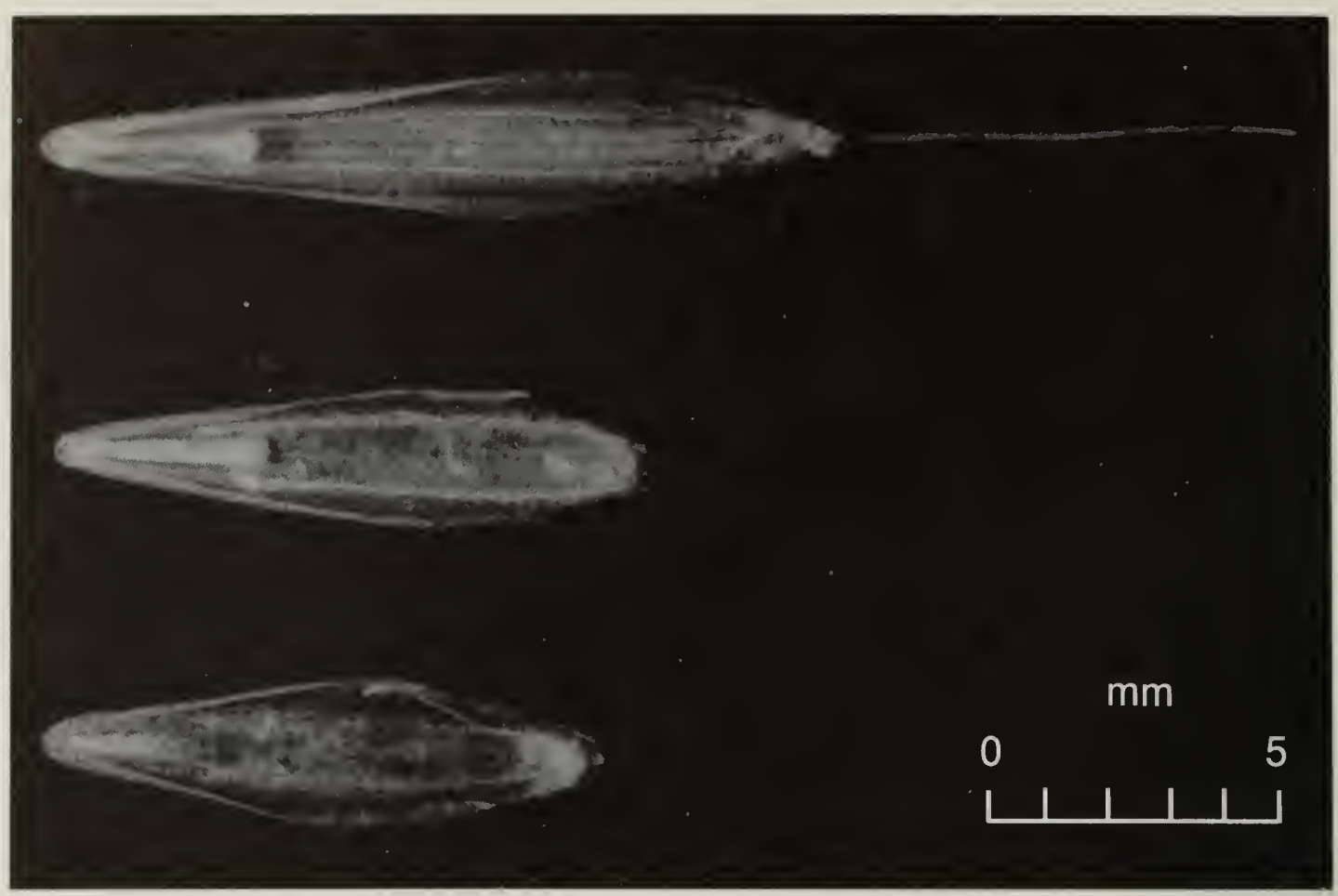

Fig. 5 Graines non traitées du brome des prés (en haut) et du brome inerme (en bas) et graines traitées du brome des prés (centre).

\section{Lectures suggérées}

Cooper, C.S.; et al. 1978. Evaluation of Regar meadow bromegrass in Montana. Montana Agric. Experiment Station, Bull. 702.

Fairey, N.A. 1991. Effects of nitrogen fertilizer, cutting frequency, and companion legume on herbage production and quality of four grasses. Can. J. Plant Sci. 71:717-725.

Knowles, R.P. 1987. Productivity of grass species in the Dark Brown soil zone of Saskatchewan. Can. J. Plant Sci. 67:719-725

Limin. A.E.; Fowler, D.B. 1987. Cold hardiness of forage grasses grown on the Canadian prairies. Can. J. Plant Sci. 67:1111-1115.

Research Highlights. 1986: Station de recherches de Melfort.

Seamands, W.J.; Kolp, B.J. 1975. Regar bromegrass. Univ. of Wyoming Agric. Extension Service, B-625. 


\section{Annexe}

Les producteurs qui désirent soumettre des échantillons de semences à des tests de dépistage du charbon de l'épi, au coût de 29 \$ (1992), doivent faire parvenir $250 \mathrm{~g}$ de semences, en précisant leur numéro d'identification de producteur semencier, au :

$\mathrm{D}^{\mathrm{r}}$ J. Sheppard

Agriculture Canada

Laboratoire de biologie des semences

Édifice 22, Services de laboratoire

Ferme expérimentale centrale

Ottawa (Ont.) K1A 0C6 
